\title{
Multi-level Service Differentiation Scheme for the IEEE 802.15.4 Sensor Networks*
}

\author{
Euijik Kim ${ }^{1}$, Meejoung Kim², Sungkwan Youm ${ }^{1}$, Seokhoon Choi ${ }^{1}$, \\ and Chul-Hee Kang ${ }^{1}$ \\ ${ }^{1}$ Department of Electronics Engineering, Korea University, \\ 1, 5-ga, Anam-dong, Sungbuk-gu, Seoul 136-701, Korea \\ \{ejkim, skyoum, shchoi, chkang\}@widecomm.korea.ac.kr \\ ${ }^{2}$ Research Institute for Information and Communication Technology, Korea University, \\ 1, 5-ga, Anam-dong, Sungbuk-gu, Seoul 136-701, Korea \\ meejkimakorea.ac.kr
}

\begin{abstract}
In the sensor networks, the data packets transmitted by the different devices in the home networking and the industrial application maintain the different levels of importance. In this paper, we propose two mechanisms for the IEEE 802.15.4 sensor networks to provide the multi-level differentiated services which are required by each and every device. The mathematical model based on the discrete-time Markov chain is presented and is analyzed to measure the performances of the proposed mechanisms. The numerical results show the effect of the variation of the contention window size and the backoff exponent for the service differentiation on the 802.15.4 sensor networks. From the results, we derive that the contention window size is more affective than the backoff exponent on the service differentiation while the backoff exponent is more affective than the contention window size on the average delay of every device. Simulation results are also given to verify the accuracy of the numerical model.
\end{abstract}

\section{Introduction}

For the last few years, the researches on the wireless sensor networks have been increased significantly. Terms such as pervasive computing and smart spaces are being used to describe the future computing and communications. These concepts are adopted to our personal and business domains being densely populated with miniature sensors, which are constantly monitoring the environment and reporting the data to each other or to some central base station. The sensor networks cover from small applications such as the health monitoring to large applications such as the environment surveillance. In other words, it can be used widely in practical applications from the home networking to the industrial application.

The recent IEEE 802.15.4 standard for the low rate wireless personal area networks is considered as one of the technology candidates for the wireless sensor networks,

* This research was supported by the MIC (Ministry of Information and Communication), Korea, under the ITRC (Information Technology Research Center) support program supervised by the IITA (Institute of Information Technology Assessment). 
since it supports small, cheap, energy-efficient devices operating on battery power that require little infrastructure to operate, or none at all $[1,2]$.

IEEE 802.11e is the research for the service differentiation. It supports the service differentiation by applying a number of queues in one node. The contention-based channel access function of IEEE 802.11e is EDCF, in which multiple queues (up to 8) are used for different priorities (up to 8). Priorities are achieved by differentiating the arbitration inter-frame space and the initial window size. However, it is not possible to use multiple queues in the sensor networks. In addition, it does not considered the high capacity traffics, which is not suitable for the sensor networks.

In this paper, we observe the data packets that are transmitted by the different devices which have different levels of importance in the sensor networks using in the home networking and the industrial application [3]. Considering the home networking, for the sensor devices performing the gate security system and the temperature sensor of the fire-warning facilities, the data packet losses for the sensor devices could be fatal. On the contrary, the data packet losses for the sensor devices which belong to TVs or refrigerators might be negligible. In addition, considering the sensor devices belong to the production line in the industrial application, each device requires the different bandwidth in a network. If there are two lines producing 30 and 60 products per second, each device in each line requires the different processing rates and the bandwidths in a network. Obviously, devices which require the high bandwidths and produce the emergency data have to take the priorities prior to other devices.

In this paper, we propose two mechanisms for the modified 802.15.4 sensor network which provide multi-level differentiated services for each and every device $[4,5]$. The proposed mechanisms make it possible to provide the superior services to the device which requests the high Quality of Service (QoS) prior to other devices. We present the mathematical model for the proposed mechanisms based on the 802.15.4 sensor networks, which is based on previous works of analyzing IEEE 802.11 [6]. We consider the beacon-enabled mode with the slotted CSMA-CA algorithm in our model and assume the saturation conditions, i.e. each and every device always has a packet waiting to be transmitted, for the performance analysis. The mathematical model is based on the discrete-time Markov chain in which each component of state is representing the situation of the head packet in the queue of a device. By analyzing the Markov chain, we obtain the access probability for the device and the probability that the medium is idle. Moreover, we obtain the saturation throughput and the saturation delay. We expect the model proposed in this paper can be a reference model for the people who produce and use the sensor devices in matters of the parameter setting.

The rest of the paper is organized as follows. In section 2, we propose the operating mechanism and the mathematical model for the proposed mechanisms is presented in section 3 . In section 4 , we evaluate the performances of the proposed mechanisms such as throughput and delay through the mathematical analysis. Section 5 provides the numerical and simulation results which show the accuracy of the proposed mechanisms. Finally, section 6 concludes the paper.

\section{Multi-level Service Differentiation Scheme}

In this section, we propose two mechanisms, differentiation by the contention window size and differentiation by the backoff exponent, to provide the service differentiation 
by multiple different level priorities in the IEEE 802.15.4 wireless sensor networks. Priority of a device is chosen through the proposed two service differentiation mechanisms and all devices are divided into multiple different priority classes. Even though it can be also considered the combined mechanism of the proposed mechanisms, we describe the mechanisms separately in this paper. Before describing the mechanisms in detail, we first state our assumptions. The 802.15.4 sensor network model that we are considering operates in beacon-enable mode with slotted CSMACA algorithm. In this paper we only consider the contention access period (CAP) and analyze the proposed mechanisms in the saturation mode. When the transmitted packet meets the collision, the packet is dropped and the device tries to transmit the new packet in the head of the queue. In the following we describe the proposed mechanisms in detail.

\subsection{Service Differentiation by Contention Window Size}

All devices within the network are divided into multiple different priority classes by differentiating the contention window size in phase. A CW value is set to CW[q] according to the priority of the device. Namely, the early stage of network forming phase, every sensor device is assigned with the different priority according to the importance of the function by differentiating the $\mathrm{CW}$ value. For example, if three priority classes, high, middle, and low, are considered, q may take the values of 0,1 , or 2 . If $\mathrm{q}=0$, then $\mathrm{CW}=\mathrm{CW}[0]$ and it means the priority 0 class (high class), while for $\mathrm{q}=1$ and $2, \mathrm{CW}=\mathrm{CW}[1]$ and $\mathrm{CW}=\mathrm{CW}[2]$ mean the priority 1 class (middle class) and the priority 2 class (low class) respectively. To guarantee the priority, the relation among the three values has to be as follows.: $\mathrm{CW}[0]<\mathrm{CW}[1]<\mathrm{CW}$ [2] Such a setting of the differentiated CW value within the 802.15.4 plays a similar role in AIFS differentiation depending on traffics within the 802.11e EDCF.

\subsection{Service Differentiation by Backoff Exponent}

In the early stage of the network forming phase, every sensor device is assigned with the different priority according to the functional importance by differentiating the backoff exponent. A BE value is set to $\mathrm{BE}[\mathrm{q}]$ according to the functional importance of the device. For example, if three priority classes, high, middle, and low, are considered, $q$ may take the values of 0,1 , or 2 . If $q=0$, then $B E=B E[0]$ and it means the priority 0 class (high class), while for $\mathrm{q}=1$ and $2, \mathrm{BE}=\mathrm{BE}[1]$ and $\mathrm{BE}=\mathrm{BE}[2]$ mean the priority 1 class (middle class) and the priority 2 class (low class) respectively. To guarantee the priority, the relation among the three values has to be as follows: $\mathrm{BE}[0]$ $<\mathrm{BE}[1]<\mathrm{BE}[2]$

The BE differentiation of the proposed mechanism differentiates the randomly chosen backoff duration. There is a difference between the backoff duration of the proposed mechanism and the backoff duration of the conventional 802.15.4 as follows.: At the beginning of the backoff stage 0 , it chooses a random value in the range of $[0$, $\left.W_{0}-1\right], W_{0}=2^{B E[q]}$, as a backoff counter. When it senses the channel becomes busy, the backoff stage of it is increased by one and the BE value is also increased by one, and the backoff counter is randomly chosen in $\left[W_{0}, W_{1}-1\right]\left(W_{1}=2 W_{0}\right)$. More details are given to the next section. 


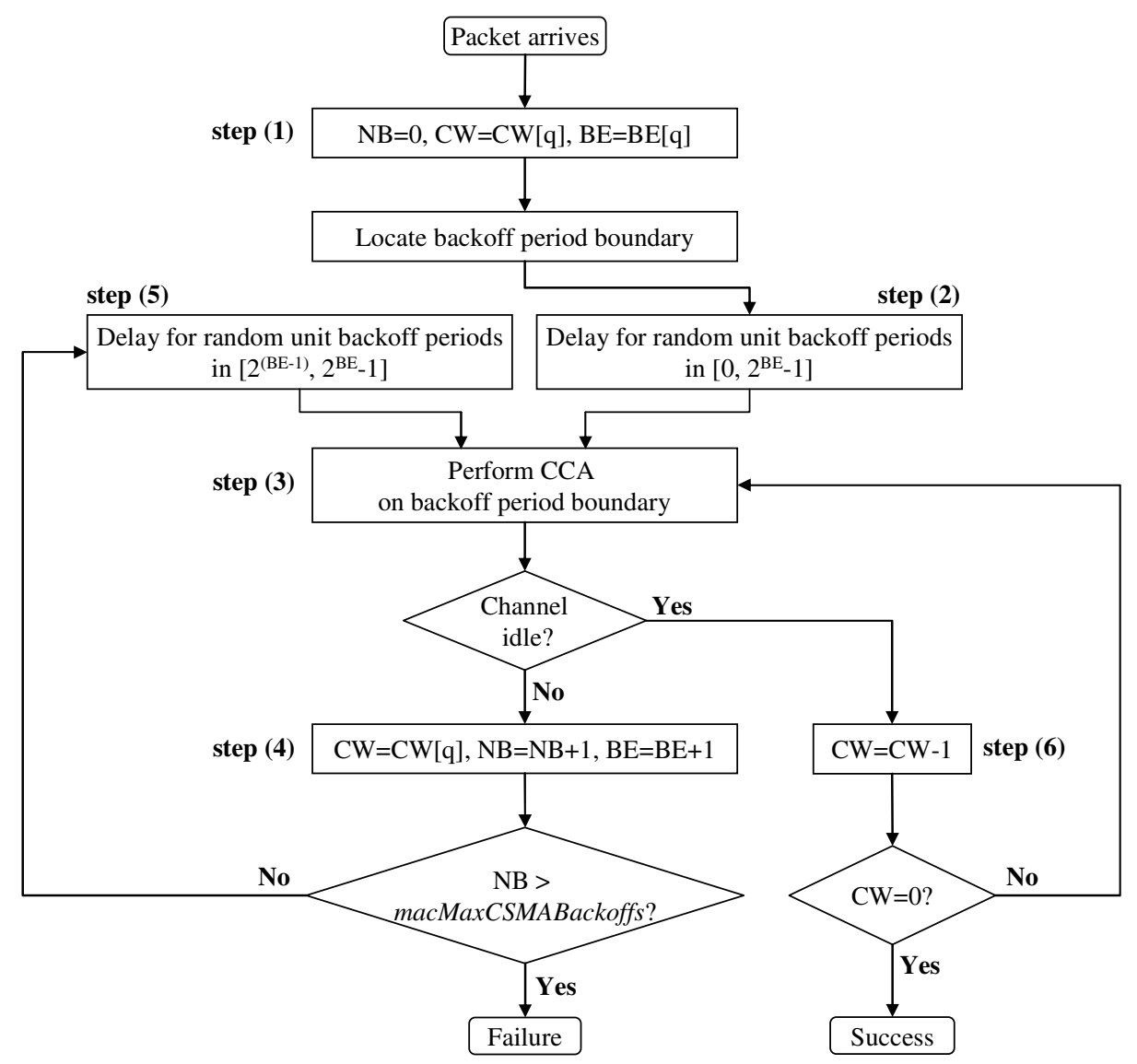

Fig. 1. The operation of the multi-level service differentiation scheme

\subsection{Operation of Multi-level Service Differentiation Scheme}

Fig. 1 describes the operation of the proposed scheme. In the figure, step (1) initialize the $\mathrm{NB}$ value as 0 and set the values of $\mathrm{CW}$ and $\mathrm{BE}$ according to the priority classes (one of the two or both of mechanisms can be selected depending on the requested service differentiation). In step (2), the algorithm attempts to avoid collisions by selecting a random waiting time in the range of $\left[0,2^{B E}-1\right]$. The MAC sublayer will proceed with step (3) and performs the first CCA (Clear Channel Assessment) to see whether the channel is idle. If the channel is busy, the values of NB and BE are increased by one and the $\mathrm{CW}$ value is set to $\mathrm{CW}[\mathrm{q}]$ again which is the same value as in the first backoff stage. If the number of retries in step (4) is less than or equal to macMaxCSMABackoffs (the default value of which is 5), the algorithm goes to step (5). In step (5), while the backoff period within the standard 802.15.4 can take any value in the range of $\left[0,2^{B E}-1\right]$, the proposed scheme can take the value in the range excluding the last backoff stage. In other words, the range of the backoff counter is 
given by $\left[2^{B E-1}, 2^{B E}-1\right]$, which is the randomly selected backoff period. Such a mechanism ensures the service of the high priority class devices prior to others by increasing the differences of the backoff period. If the values of NB is above macMaxCSMABackoffs, the algorithm terminates with a channel access failure status. The failure will be reported to the higher protocol layers. On the other hand, if the channel is idle, the procedure goes to step (6). In other words, the CW value is decreased by one and the channel is assessed again. When the channel is idle in subsequent slots and so the $\mathrm{CW}$ value becomes 0 , the packet transmission begins, provided the remaining number of backoff periods in the current superframe suffices to handle both the packet and the subsequent acknowledgment. Whether the transmission succeeds or fails, the algorithm goes to the next packet of the queue of the device.

\section{Analytical Model}

To analyze the proposed scheme, we introduce the following three random variables for a given device in the priority $q$ class. Let $n(q, t)$ and $c(q, t)$ be the stochastic processes representing the values of $\mathrm{NB}$ and $\mathrm{CW}$, respectively at time $t$. Let $b(q, t)$ be the stochastic process representing the value of the backoff counter. Note that NB represents the backoff stage within the range of $[0, m+1], m=$ macMacCSMABackoffs, and $q$ gives the multiple priorities taking values in $[0, \infty)$.

The process $\{n(q, t), c(q, t), b(q, t)\}$ forms a multi-dimensional Markov process defining the state of the packet at the backoff unit boundaries. Since every and each device has its own priority which does not change, the process $\{n(q, t), c(q, t), b(q, t)\}$ can be written simply as $\{n(t), c(t), b(t)\}$. Then the corresponding state space is denoted as follows:

$$
\Omega=\left\{(n(t), c(t), b(t)) \mid 0 \leq n(t) \leq m+1,0 \leq c(t) \leq q, 0 \leq b(t) \leq W_{i}-1, i=0, \cdots, m\right\}
$$

where $W_{0}=2^{B E[q]}$ and $W_{i}=2^{i} W_{0}$.

The state transition diagram of these states is illustrated in Fig. 2. For simplicity of the notations, we use the transition probabilities $P(i, j, k-1 \mid i, j, k)$ instead of $P(n(t+1)=i, c(t+1)=j, b(t+1)=k-1 \mid n(t)=i, c(t)=j, b(t)=k)$.

For analysis, we consider a fixed number of devices $n$ with each of them is always having a packet available for transmission, i.e. saturation mode. We assume that every device is classified into 3 priority classes according to its priority consisting of $n_{0}, n_{1}$, and $n_{2}$ devices satisfying $n_{0}+n_{1}+n_{2}=n$. Then the one-step transition probabilities are given as follows.:

$$
\begin{aligned}
& P(0, q, k \mid i, 0,0)=1 / W_{0}, \quad \text { for } i \in[0, m], k \in\left[0, W_{0}-1\right] \\
& P(0, q, k \mid m+1,0,0)=1 / W_{0}, \quad \text { for } k \in\left[0, W_{0}-1\right] \\
& P(i, q, k-1 \mid i, q, k)=1, \quad \text { for } i \in[0, m], k \in\left[1, W_{i}-1\right] \\
& P(i, j-1,0 \mid i, j, 0)=p_{I}, \quad \text { for } i \in[0, m], j \in[1, q] \\
& P\left(i+1, q, W_{i-1}+k \mid i, j, 0\right)=\left(1-p_{I}\right) / W_{i-1}, \quad \text { for } i \in[0, m], j \in[1, q], k \in\left[0, W_{i-1}-1\right]
\end{aligned}
$$




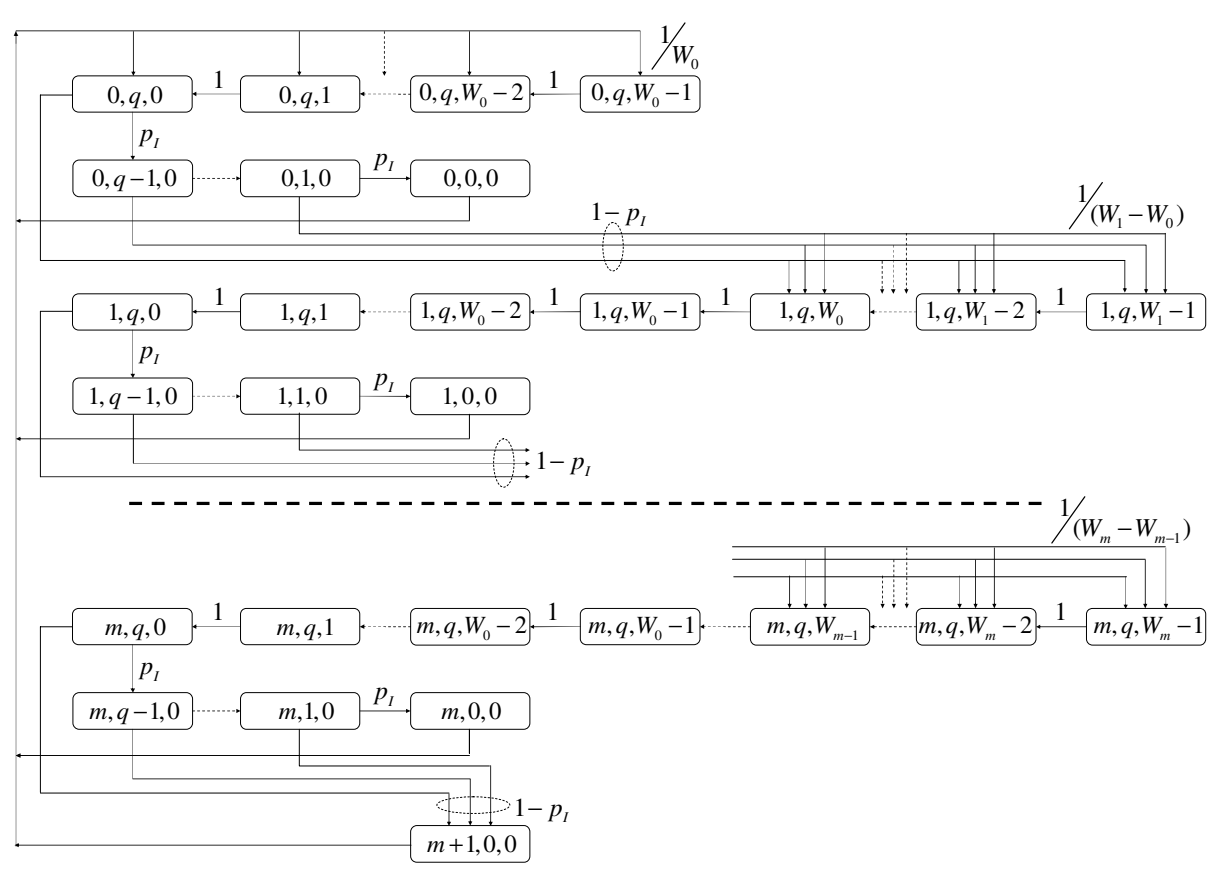

Fig. 2. Markov chain model

Note that the states are positive recurrence and the system is stable. Therefore, there exist the stationary probabilities of the discrete-time Markov chain which is defined by $b_{i, j, k}=\lim _{t \rightarrow \infty} P(n(t)=i, c(t)=j, b(t)=k)$, for $i \in[0, m+1], j \in[0, q]$, and $k \in\left[0,2^{B E}-1\right]$. Let $\mathbf{b}$ be the stationary vector, i.e.,

$$
\mathbf{b}=\left(b_{0,0,0}, b_{0,1,0}, \cdots b_{0, q, W_{0}-1}, \cdots, b_{m, 0,0}, \cdots, b_{m, q, W_{m}-1}, b_{m+1,0,0}\right) .
$$

Then it satisfies the following two equations:

$$
\mathbf{b P}=\mathbf{b} \text { and } \mathbf{b e}=1,
$$

where $\mathbf{e}$ is the column vector whose components are consisted with 1 and $\mathbf{P}$ is the transition probability matrix when $\Omega$ is ordered lexicographically. By using the first part of Eq. (1), we obtain the following relations between stationary probabilities.:

$$
\begin{gathered}
b_{i, 0,0}=b_{0,0,0}\left(1-p_{I}^{q}\right)^{i}, \quad i \in[0, m] \\
b_{i, q, k}=b_{0,0,0}\left(1-p_{I}^{q}\right)^{i+1} / p_{I}^{q}, \quad i \in[1, m] ; k \in\left[0, W_{i-1}-1\right] \\
b_{i, q, W_{i-1}+k}=b_{0,0,0}\left\{\left(W_{i-1}-k\right)\left(1-p_{I}^{q}\right)^{i+1} / W_{i-1} p_{I}^{q}\right\}, \quad i \in[1, m] ; k \in\left[0, W_{i-1}-1\right] \\
b_{0, q, k}=b_{0,0,0}\left\{\left(W_{0}-k\right) / W_{0} p_{I}^{q}\right\}, \quad k \in\left[0, W_{0}-1\right] \\
b_{i, j, 0}=b_{0,0,0} p_{I}^{j}\left(1-p_{I}^{q}\right)^{i}, \quad i \in[0, m] ; j \in[1, q-1] \\
b_{m+1,0,0}=b_{0,0,0}\left(1-p_{I}^{q}\right)^{m+1} / p_{I}^{q}
\end{gathered}
$$


We obtain $b_{0,0,0}$ by substituting Eq. (2) into the second part of Eq. (1). By substituting $b_{0,0,0}$ into each equation in Eq. (2), we can obtain the stationary probabilities $b_{i, j, k}$. Then, $b_{i, 0,0}$ is derived as follows.:

$$
b_{i, 0,0}=\frac{2 p_{I}^{q}\left(1-p_{I}^{q}\right)^{i}}{3-2\left(1-p_{I}^{q}\right)^{m+1}+\frac{3 W_{0}\left(1-p_{I}^{q}\right)^{2}\left(1-2^{m}\left(1-p_{I}^{q}\right)^{m}\right)}{2 p_{I}^{q}-1}+\frac{2\left(1-p_{I}^{q}\right)^{m+1}\left(p_{I}^{q}-2 p_{I}^{q}+1\right)+2 p_{I}^{q}\left(1-p_{I}^{q-1}\right)}{1-p_{I}}}
$$

With these stationary probabilities, we find the probability that the device transmits a packet at the boundary of a backoff period which will be denoted by $\tau$. Let $\tau_{q}$ be the probability that a device in the priority $q$ class transmits during a generic slot time. Then we have

$$
\tau_{q}=\sum_{i=0}^{m} b_{i, 0,0}
$$

Since the device belongs to the different priority classes has a different $\mathrm{CW}[\mathrm{q}]$ value or $\mathrm{BE}[\mathrm{q}]$ value, the index $q$ of $\tau$ is needed to differentiate the priority classes. Therefore, during the CCA procedure, the probability that the channel is idle is given by

$$
p_{I}=\left(1-\tau_{0}\right)^{n_{0}}\left(1-\tau_{1}\right)^{n_{1}}\left(1-\tau_{2}\right)^{n_{2}}, n=n_{0}+n_{1}+n_{2} .
$$

By substituting Eq. (4) to Eq. (3), we can solve unknown parameters, $\tau_{0}, \tau_{1}$, and $\tau_{2}$, numerically and then calculate $p_{I}$ from Eq. (4).

\section{Performance Analysis}

\subsection{Throughput}

Let $p_{S, q}$ denote the probability that a successful transmission occurs in a time slot for the priority $q$ class. Then these probabilities are calculated as follows.:

$$
p_{S, q}=n_{q} \tau_{q}\left(1-\tau_{q}\right)^{n_{q}-1} \prod_{h=0, h \neq q}^{2}\left(1-\tau_{h}\right)^{n_{h}}=\frac{n_{q} \tau_{q}}{1-\tau_{q}} p_{I}, \quad \text { for } q=0,1,2
$$

Let $p_{B}$ be the probability that the channel is sensed busy in a time slot. Then it is given by

$$
p_{B}=1-p_{I}=1-\left(1-\tau_{0}\right)^{n_{0}}\left(1-\tau_{1}\right)^{n_{1}}\left(1-\tau_{2}\right)^{n_{2}} .
$$

Therefore, the probability that a collision occurs in a time slot for the priority $q$ class which is denoted by $p_{C, q}$ is given by

$$
p_{C, q}=1-\left(1-\tau_{q}\right)^{n_{q}-1} \prod_{l \in Q, l \neq q}\left(1-\tau_{l}\right)^{n_{l}}, \quad \text { for } q=0,1,2 \text {. }
$$


Let $S_{q}$ be the normalized throughput for the priority $q$ class. Let $\delta, L, T_{S, q}$, and $T_{C, q}$ denote the duration of an empty time slot, the payload size, the average time that the channel is sensed busy because of a successful transmission for the priority $q$ class, and the average time that the channel has a collision for the priority $q$ class, respectively. Now we can express the normalized throughput $S_{q}$ as the following ratio:

$$
\begin{aligned}
S_{q} & =\frac{E(\text { payload information transmitted in a slot time for the priority } q \text { class })}{E(\text { length of a slot time })} \\
& =\frac{p_{S, q} E(L)}{p_{I} \delta+p_{S, q} T_{S, q}+\left(p_{B}-p_{S, q}\right) T_{C, q}}
\end{aligned}
$$

where $T_{H}, T_{E(L)}, t_{A C K}, S I F S, L^{*}, T_{E\left(L^{*}\right)}$, and $\gamma$ denote the time to transmit the header (including MAC header, PHY header), the time to transmit the payload, the time to transmit the ACK, the time of SIFS, the length of the frame in a collision, the time to transmit a payload with length $E\left(L^{*}\right)$, and the time of the propagation delay, respectively. Note that $T_{S, q}$ and $T_{C, q}$ are given by

$$
T_{S, q}=T_{H}+T_{E(L)}+2 S I F S+\gamma+t_{A C K}
$$

and

$$
T_{C, q}=T_{H}+T_{E\left(L^{*}\right)}+2 S I F S+\gamma+t_{A C K}
$$

\subsection{Average Delay}

In this paper, the delay of a packet is defined as the time elapsed from the instant of the generation of the packet to the instant of the successful reception or drop of it. Let $D$ be the time to process the transmission. Note that $D$ is a random variable depending on the priority $q$. Let $E(D)$ be the mean value of $D$. In the discrete-time Markov chain model of section 4 , for each state $(i, j, k)$, the mean value of the delay $E\left(D_{i, j, k}\right)$ can be presented as follows:

$$
\begin{aligned}
& E\left(D_{i, 0,0}\right)=\delta, \quad \text { for } i \in[0, m+1] \\
& E\left(D_{i, q, k}\right)=\delta k+\delta E\left(D_{i, q, 0}\right), \quad \text { for } i \in[0, m] ; k \in\left[0, W_{i}-1\right] \\
& E\left(D_{i, j, 0}\right)=\delta p_{I}\left(1+E\left(D_{i, j-1,0}\right)\right)+\delta \sum_{k=1}^{W_{i}} \frac{1-p_{I}}{W_{i}}\left(1+E\left(D_{i+1, q, W_{i+1}-k}\right)\right), \\
& \text { for } i \in[0, m-1] ; j \in[1, q] \\
& E\left(D_{m, j, 0}\right)=\delta \sum_{r=1}^{j}\left(p_{I}^{j-r}+p_{I}^{j-2}\right), \quad \text { for } j \in[2, q] \text {. }
\end{aligned}
$$

Therefore, the mean of total delay can be found by the following equation.

$$
E\left(D_{\text {total }}\right)=\delta \sum_{i=0}^{m} \sum_{j=0}^{q} \sum_{k=0}^{W_{i}-1} E\left(D_{i, j, k}\right) b_{i, j, k}+\delta E\left(D_{m+1,0,0}\right) b_{m+1,0,0}
$$




\section{Numerical and Simulation Results}

In this section we present the performance of the analysis and compare the numerical and simulation results to verify the accuracy of the proposed numerical model. The numerical results show the effect of the variations of $\mathrm{CW}[\mathrm{q}]$ and $\mathrm{BE}[\mathrm{q}]$ for the service differentiation. Simulations are performed with a simple Matlab simulator. The parameters used in the numerical analysis and the simulation refer to the BPSK mode and are listed in Table 1. Also some assumptions are made for simplifying the simulation and the numerical analysis without losing the comprehensive analysis of the model. These assumptions can be accepted in the saturation mode we consider in this paper. We assume that the size of packets is constant. In addition, we assume that the required time to transmit a packet whether it succeeds or fails is the total time to transmit a data and an ACK packet with 2 SIFS intervals so that ACK packets are never lost.

In order to verify the accuracy of the proposed model, the comparison of throughputs with a varying number of devices within the each class is presented in Table 2. The value of CW[q] is set by 2 for all the devices in every class and the values of $\mathrm{BE}[0], \mathrm{BE}[1]$, and $\mathrm{BE}[2]$ at the device of each class are set by 2, 3, and 4, respectively. As shown in Table 2, the results of simulation are almost the same as those of numerical analysis. All simulation results in the table are obtained with a 98\% Confidential Rate. In the tables, the Confidential Rate $(C R)$ between numerical and simulation results are given, which is calculated by the following equation.:

$$
C R=\left(1-\left|\frac{E\left[S_{\text {num }}-S_{\text {sim }}\right]}{E\left[S_{\text {num }}\right]}\right|\right) \times 100 \%
$$

Moreover, both results of the numerical analysis and the simulation show the throughput at a class is almost twice as that at the class of one level lower priority. Since the difference between the numerical and simulation results is negligible, in the remained figures we present the numerical results only.

Table 1. The parameter set used in the numerical analysis and simulation

\begin{tabular}{|l|l|l|l|}
\hline Packet payload & 816 bits & Channel bit rate & $20 \mathrm{Kbit} / \mathrm{s}$ \\
\hline ACK & 40 bits & SIFS & 12 symbols \\
\hline MAC header & 200 bits & Retry limit & 5 \\
\hline PHY header & 48 bits & Slot time & 20 symbols \\
\hline
\end{tabular}

Table 2. Comparison of throughputs with varying number of devices

\begin{tabular}{|c|c|c|c|c|c|c|c|c|}
\hline \multicolumn{2}{|c|}{$\begin{array}{c}\text { The number of node for } \\
\text { each class }\end{array}$} & \multicolumn{3}{|c|}{ Analysis } & \multicolumn{3}{c|}{ Simulation } \\
\hline High & Middle & Low & High & Middle & Low & High & Middle & Low \\
\hline 5 & 5 & 5 & $3,621.8$ & $1,782.0$ & 883.96 & $3,645.6$ & $1,781.2$ & 881.36 \\
\hline 10 & 10 & 10 & $2,514.4$ & $1,236.2$ & 612.95 & $2,522.4$ & $1,213.7$ & 612.45 \\
\hline 15 & 15 & 15 & $1,971.6$ & 971.3 & 482.07 & $1,977.5$ & 972.5 & 482.47 \\
\hline 20 & 20 & 20 & $1,653.7$ & 816.1 & 405.39 & $1,654.8$ & 817.3 & 401.23 \\
\hline 25 & 25 & 25 & 1,420 & 701.8 & 348.87 & 1,423 & 702.6 & 348.47 \\
\hline
\end{tabular}




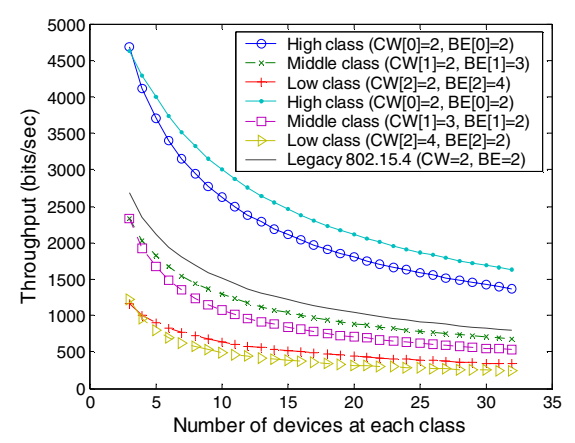

Fig. 3. Comparison of throughput

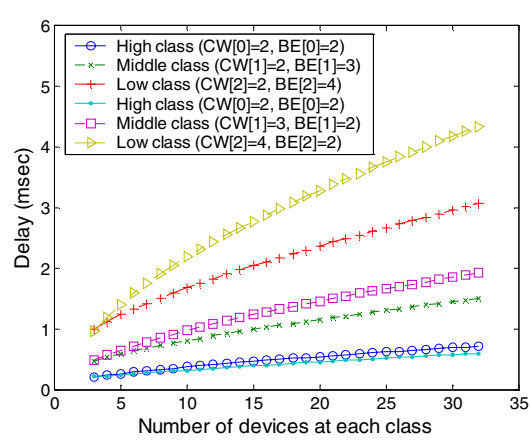

Fig. 4. Comparison of delay

The saturation throughputs for the varying values of $\mathrm{CW}[\mathrm{q}]$ and $\mathrm{BE}[\mathrm{q}]$ are shown in Fig. 3. In the figure, $x$ and $y$ axis denote the number of devices at each class and the throughput, respectively. We assume the number of devices in each class is all the same in the figures of numerical results. In other words, if $\mathrm{x}$ is 5 , there is the total number of 15 devices. We analyze the effect of the variation of parameters CW[q] and $\mathrm{BE}[\mathrm{q}]$ for a varying number of devices. The throughput of the legacy 802.15.4 is equal to the average throughput of the three classes. The throughput of high classes at the variation of $\mathrm{CW}[\mathrm{q}]$ or $\mathrm{BE}[\mathrm{q}]$ outperforms than that of the legacy 802.15.4 while the throughput of middle and low classes is degraded below that of the legacy 802.15.4. The throughput of a device in each class decreases as the total number of devices increases which dues to the fact that the device has to run the backoff algorithm more since more devices will lead more collision. The throughput of high class from results set with the variation of $\mathrm{CW}[\mathrm{q}]$ is higher than that from results set with the variation of $\mathrm{BE}[\mathrm{q}]$. The increment of $\mathrm{CW}[\mathrm{q}]$ means that the devices of lower class yield the opportunity to occupy the channel to the devices in higher class. Therefore, the probability of retrying the transmissions of a device in lower class increases as the value of $\mathrm{CW}[\mathrm{q}]$ at the class increases. The delicate tuning of the throughput could be performed by varying the value of $\mathrm{BE}[\mathrm{q}]$ while the value of CW[q] could be adjusted to increase the throughput of the high class.

Fig. 4 shows that the saturation delay increases almost linearly as the number of devices increases. The delay of the low class is larger than those of any other classes with respect to the variation of $\mathrm{CW}[\mathrm{q}]$ and $\mathrm{BE}[\mathrm{q}]$. The characteristic of the saturation delay is that there are differences between the delay summations of all classes with the variation of $\mathrm{CW}[\mathrm{q}]$ and that of $\mathrm{BE}[\mathrm{q}]$. In other words, the delay summations of all the classes with the variation of $\mathrm{BE}[\mathrm{q}]$ is smaller than that with the variation of $\mathrm{CW}[\mathrm{q}]$. Therefore, to minimize the saturation delay, it is better to vary $\mathrm{BE}[\mathrm{q}]$ rather than $\mathrm{CW}[\mathrm{q}]$.

\section{Conclusion}

In this paper, we proposed two mechanisms for the IEEE 802.15.4 sensor networks which provide the multiple level differentiated services for each and every device. The mathematical model based on the discrete-time Markov chain is provided for analyzing the performance of the proposed mechanisms. 
The comparison of numerical and simulation results is given to verify the accuracy of the numerical model. The numerical results of several performance measures are given to analyze the effect of the variation of the contention window size and the backoff exponent for service differentiation on the 802.15.4 sensor networks. Increasing the contention window size or the backoff exponent within a class means that the devices of lower class yield an opportunity to occupy the channel prior to those of higher class. Therefore, the probability to retry a transmission at lower class increases, which dues to a busy channel, as the contention window size or the backoff exponent at the class increases. The numerical results show that the variation of the contention window size has more effect on the service differentiation than that of the backoff exponent even though the average delay of every device is affected more by the backoff exponent. The delicate tuning of the throughput could be performed by varying the backoff exponent, while the increase of the throughput of the high class could be performed by adjusting the contention window size.

The results obtained in this paper provide a criterion for using the parameters for specific purposes. Therefore, we expect the proposed model can be a reference model for the people who produce and use the sensor devices in matters of the parameter setting.

\section{References}

1. Standard for part 15.4, Wireless medium access control (MAC) and physical layer (PHY) specifications for low rate wireless personal area networks (WPAN), IEEE Std 802.15.4, IEEE, New York, NY. (2003)

2. Misic, J., Shafi, S., Misic, V.B.: The Impact of MAC Parameters on the Performance of 802.15.4 PAN, Elsevier Ad hoc Networks, Vol. 2. (2004) 351-371

3. Bhatnagar, S., Deb, B., Nath, B.: Service Differentiation in Sensor Networks, 4th International Symposium on Wireless Personal Multimedia Communications, Vol. 1. (2001) 435-441

4. Robinson, J.W., Randhawa, T.S.: Saturation Throughput Analysis of IEEE 802.11e Enhanced Distributed Coordination Function, IEEE Journal on Selected Areas in Communications, Vol. 22. (2004) 917-928

5. Xiao, Y.: Enhanced DCF of IEEE 802.11e to Support QoS, IEEE Wireless Communications and Networking Conference, Vol. 4. (2003) 1291-1296

6. Bianchi, G.: Performance Analysis of the IEEE 802.11 Distributed Coordination Function, IEEE Journal on Selected Areas in Communications, Vol. 18. (2000) 535-547 